\title{
Microcore: A Playable Case Study for Improving Adolescents' Argumentative Writing in a Workplace Context
}

\author{
Derek L. Hansen \\ Brigham Young University \\ dlhansen@byu.edu
}

\author{
Jonathan Balzotti \\ Brigham Young University \\ jonathan balzotti@,byu.edu
}

\author{
Lauren Fine \\ Brigham Young University \\ finelauren1@gmail.com
}

\author{
Daniel Ebeling \\ Brigham Young University \\ de6eling@gmail.com
}

\begin{abstract}
A playable case study is an immersive, transmedia story controlled by a puppetmaster (i.e., teacher), but played by participants who advance the plot through their contributions and interactions with fictional characters. They are also explicitly educational, consisting of both the immersive, transmedia story, as well as in-game and out-of-game materials provided for educational scaffolding and reflection. We present the Microcore playable case study to illustrate the potential of this new type of experiential simulation that incorporates aspects of Alternate Reality Games (ARGs) to increase immersion. We present results from a pilot test of Microcore with an undergraduate course, identifying design strategies that worked well and others that led to improvements that are currently being incorporated. We also provide questions to prompt future designers of playable case studies and discuss our findings in a broader context of educational simulations.
\end{abstract}

\section{Introduction}

Driven to increase student literacy skills, expose readers to increasingly difficult texts, and prepare students for the demands of the $21^{\text {st }}$ century workforce, state and national standards require secondary education teachers to teach more sophisticated writing, reading, speaking and listening skills than ever before [1]. Despite recent efforts at the secondary and collegiate level, the workplace readiness of the Millennial generation is dismal. Over $50 \%$ of those who hire recent high school graduates found their overall preparation deficient, and less than a quarter of those who hired undergraduates rated them as having excellent basic knowledge and applied skills [2] Indeed, the strong emphasis on standardized testing of core math and reading skills, often divorced from realworld contexts, has detracted from important workforce readiness skills.

These include metacognitive skills such as problem solving when facing unknown scenarios, critical thinking when evaluating the quality of information, and metacognition when appraising the accuracy of one's own memory [3]. Additionally, 77\% of employers see soft skills (less tangible skills such as a positive attitude, dependability, team-oriented, well organized, effective communicator, and flexible) as being equally important as hard skills [4].

There are few opportunities to develop these metacognitive and soft skills in real-world contexts during traditional classroom instruction. They are best taught through "experiential learning" where learning occurs through the process of applying knowledge and conceptual understanding to real-world problems [5]. A number of interactive learning techniques, ranging from case studies to simulations to virtual labs to serious games, have been used to support experiential learning. A growing body of literature has examined these various genres of interactive learning, along with their opportunities and limitations [6-11]. However, there is considerable room for new types of technology-mediated, interactive learning, especially in the area of workforce literacy.

The goal of this project was to develop an immersive, transmedia simulation designed to prepare adolescent students to apply critical thinking and argumentative writing skills in a workplace context. We call it the Microcore Playable Case Study, or "Microcore" for short. While hundreds of educational simulations and serious games have been created, we could not identify any that focus specifically on writing skills in a workplace context. Furthermore, our specific type of "experiential simulation" [12] utilizes several novel techniques inspired by Alternate Reality Games (ARGs), which we describe and evaluate. While a fullscale evaluation of Microcore still remains, our design rationale and pilot study results are presented here to help inspire future playable case studies that can leverage similar techniques. In short, we address the following research questions in this paper:

- What techniques can be used to create an authentic and immersive experiential simulation - i.e., a playable case study?

- How can a playable case study support the development of argumentative writing skills in a simulated workplace context? 


\section{Background}

There is a long history of educational simulations, beginning in the late $1950 \mathrm{~s}$ and continuing to recent times. Gredler identifies simulations as including (a) a complex real-world situation, (b) a defined role for a participant to interact with that situation, (c) a rich environment that allows participants to execute a range of strategies, and (d) feedback for participant actions in the form of changes in the problem or situation [13]. He also differentiates between two types of educational simulations, one of which is "experiential simulations," which: "establishes a particular psychological reality and places the participants in defined roles within that reality. The participants, in the context of their roles, execute their responsibilities in an evolving situation. Experiential simulations, in other words, are dynamic case studies with participants on the inside" [12].

This description of a participant "on the inside" of a complex, unfolding real-world narrative that they can influence, sounds a lot like a player of an Alternate Reality Game (ARG). ARGs are a relatively new genre of transmedia storytelling in which "players collaboratively hunt for clues, make sense of disparate information, and solve puzzles to advance an everchanging narrative that is woven into the fabric of the real world" [14]. Players of ARGs subscribe to the "this is not a game" (TINAG) ethos, wherein they participate in the experience in authentic ways that make it feel like it is not a game, although in most cases they know it is. ARGs are also told through the use of a variety of media channels, wherein players may watch videos from fictional characters, hack into fictional company websites, and even interact via messaging or email or even payphones with fictional characters and other players. Finally, players can influence the unfolding narrative, which is ultimately controlled and modified by the "puppetmasters" (i.e., those running the game, also called the gamerunners).

Originally created for entertainment and marketing purposes, their potential as an educational platform has begun to be explored [15-18]. While the authentic nature of ARGs provides many learning opportunities, most ARGs run a single time and are not replayable, making them costly to produce and limiting their potential reach [14]. Strategies for creating reusable ARGs are being identified [19], and some of them look very much like a more recent incarnation of experiential simulations.

Against this backdrop, we propose the term "Playable Case Study" to describe an experiential simulation that leverages ARG techniques including the transmedia narrative, "this is not a game" ethos, and responsiveness of a "puppetmaster." We know of no experiential simulations that use these techniques and believe that their incorporation into a simulation warrants the new name for the experiential simulation. In short, a playable case study is an immersive, transmedia story controlled by a puppetmaster, but played by participants who advance the plot through their contributions and interactions with fictional characters. They are also explicitly educational, consisting of both the immersive, transmedia story, as well as in-game and out-of-game materials provided for educational scaffolding and reflection.

\section{Methods}

We used a mixed-methods approach to explore the potential for playable case studies on learning argumentative writing in a workplace context. The project moved in three steps: (1) We created one playable case study, Microcore, including related media and technology artifacts (i.e., user interface, video narratives, media content, instructor backend, and educational materials); (2) We deployed Microcore within a single college technical writing course with 25 students and 1 instructor and collected feedback from the beta-testers; and, (3) We updated our designs of Microcore based on the feedback received. Our final step will include a full evaluation of Microcore in secondary education writing courses taught to upperlevel High School students. Although this full evaluation has not been conducted, the lessons learned so far warrant sharing with the wider education and user experience design communities. Below is a more detailed description of the completed phases.

Step One: Microcore was developed as a close collaboration between three faculty members at [anonymized] University, along with an interdisciplinary team of students with backgrounds in education, technical and creative writing, user experience design, information technology, and media production. One of the faculty teaches a technical writing course, which is one potential outlet for our simulation, while another has extensive experience in High School writing settings. We began by identifying key argumentative writing and workplace writing learning outcomes and designing activities related to them. Next, an iterative development process was used, wherein low-fidelity prototypes (i.e., simplified mockups of user interface and learning activities) were created and tested with potential users, updated, and eventually were replaced with a high-fidelity prototype (i.e., fully functional, immersive experience).

Step Two: The research team identified an introductory-level technical writing course at [anonymized] University and an instructor willing to use the Microcore simulation in Fall 2015. There were 25 students in the course, who were Juniors and Seniors from a variety of majors that require the technical writing course. The instructor had extensive 


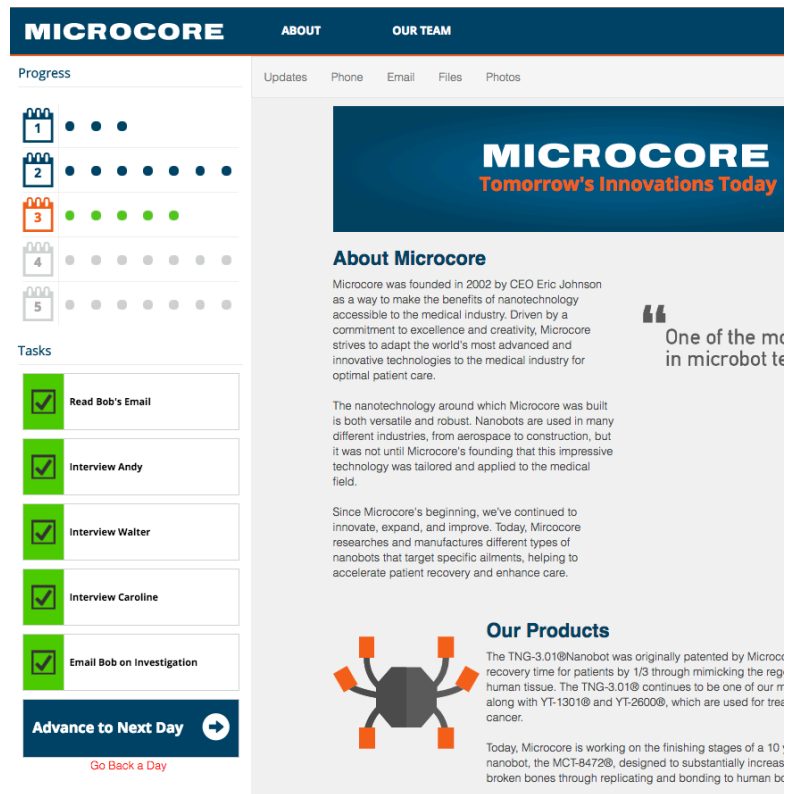

Figure 1. Microcore fictional About page with simulation days 1 through 5 indicated in the upper-left and tasks for the current day (Day 3) on the bottom-left.

experience teaching with traditional case studies, but not with this new playable case study or any other educational simulations. Our team members met with him to make sure he understood the technical details, as well as the main assignments and functionality of the simulation. He was encouraged to ask questions if needed. The simulation was discussed in class, similar to how case studies are discussed, with the online portion completed as homework. Assignments submitted in the simulation (over a 2 week period) received course grades.

Three sources of data were collected from the participants: observation notes taken during classtime, a class-wide focus group, and an instructor interview. The focus group and interview were recorded for later analysis. All data was collected with permission from the students and with proper Institutional Review Board (IRB) approval. The focus group asked students to discuss some of the positive and negative aspects of the Microcore simulation and recommendations for improvement of the user experience, assignments, story content, and other aspects of the Microcore simulation. They also asked about the benefits of the simulation, particularly as it related to writing and application of professional skills. We also interviewed the instructor using a semi-structured interview protocol that included questions about his experience teaching with the Microcore simulation and his perceptions of the students' experiences.
Data was analyzed for general themes related to learning, student motivations, user experience design, and how students judged the authenticity of the experience. We did not have preconceived codes within these categories. Instead, we let the major themes emerge from the data itself. This analysis helped us understand the needs and perceptions of the participants. All quotes provided in the paper are direct quotes from the stated sources.

Step Three: After our pilot study, we have improved the simulation. This has included extensive work on mapping assignments and experiences in the simulation to High School learning outcomes and standards at a more detailed level specific to a certain grade, as well as improvements to the user experience and educational scaffolding. In this paper, we present our most recent version, explaining elements that were changed as a result of feedback from our pilot testers. Although not all of these new features have been implemented and evaluated, we believe they are important to share since they allow us to better understand the potential of playable case studies.

\section{Microcore Playable Case Study}

This section introduces Microcore, focusing on how it incorporates the core principles of ARGs into its design. The current version is presented here, which reflects some of the recommendations based on our evaluation, which are discussed in later sections. Microcore centers around seven key Common Core learning outcomes related to argumentative writing in the English Language Arts Standards, including outcomes from writing (CCSS-ELA-LITERACY.W.910.1 and W.9-10.4), reading informational text (RI.910.6 and RI.9-10.8), literature (L.9-10.6), and speaking and listening (SL.9-10.2 and SL.9-10.3). For example, it provides opportunities for students to develop the ability to: "Produce clear and coherent writing in which the development, organization, and style are appropriate to task, purpose, and audience" (W.9-10.4) within a workplace context. Other outcomes focus on listening for an author's point of view, identifying and evaluating evidence, using valid reasoning, and other elements important to argumentative writing.

Students begin the simulation by logging into Microcore's intranet where they are greeted by Bob, a quirky but friendly intern supervisor. Bob introduces them to the functionality of the site and points out the tasks they will be assigned each day, which they check off on the left-hand side (see Figure 1). Once a day's tasks are completed, the student is allowed to advance to the next day of the simulation where Bob introduces the next set of tasks. When the simulation advances, new materials such as emails, voicemails, and files are released into the intranet site. This timed release allows 


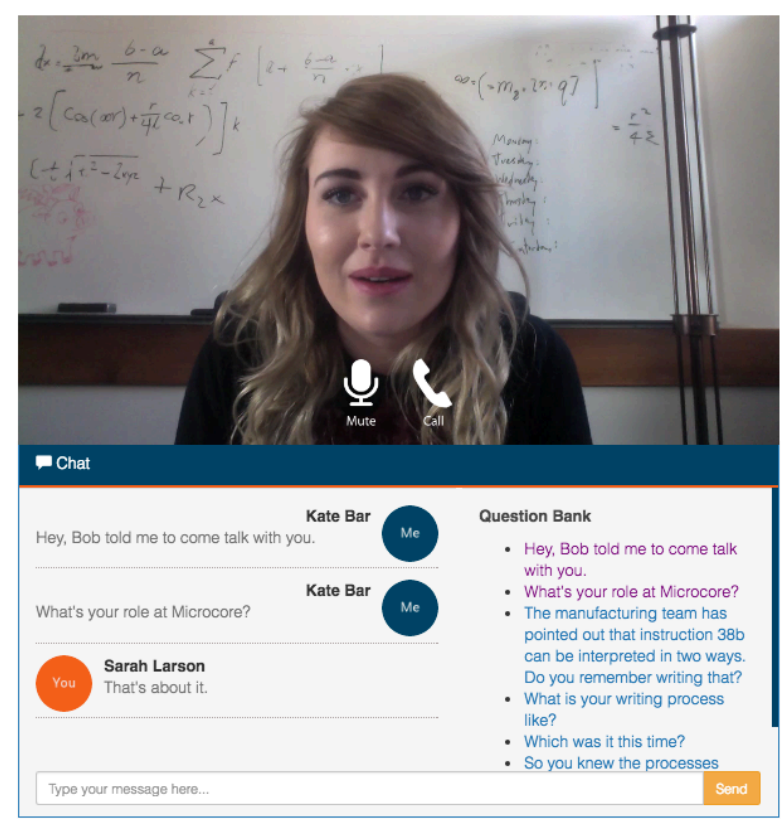

Figure 2. Video Call interface where a player (Kate Bar) is video conferencing with Sarah Larson (a fictional character).

the story to unfold as players complete assignments. Teachers can assign a simulation "day" as a homework assignment due on a specific calendar day.

This highly structured day/task structure and sidebar helps students keep track of progress and gauge what remains to be done, without disrupting the authentic feel of a corporate intranet. Specific tasks include activities such as perusing the company website to learn more about Microcore and its team, video calling team members, reading and responding to email, downloading and uploading files, and exploring photos relevant to the unfolding narrative. Each day, Bob leaves a video message explaining the tasks for the day. Although it is bounded within a single site, the variety of media channels that are used makes it a truly transmedia experience, similar to an ARG. While not implemented yet, we plan to allow players to opt in to receive messages from characters that will go directly to players' phones (e.g., text message reminders for assignments) or actual email inboxes.

All Microcore interface elements were designed to give a sense of immersion and allow players to buy into the experience, similar to how ARG players ascribe to the "This is Not a Game" ethos. While using the Microcore site, everything is "in game," including the tutorial by Bob that shows how to use the site, the assignment of tasks, and the assignment submissions (e.g., emails and files sent to characters). Additionally, we tried to make the experience as interactive as possible, recognizing that we did not have live actors available, since that would make it too costly and reduce scalability. To help with this, we created a fake video-conferencing system to allow players to "interview" and talk to characters. While all video is pre-filmed video scenes, the interface is designed to allow players to feel like they are asking questions in a live event (see Figure 2). Players click on a question from their "question bank," which is submitted as a text message with their username next to it, and the pre-filmed video jumps to the right point to answer it. A text message of the question is also sent to indicate that the question was asked. While questions are asked, characters look at the camera and patiently wait as if being spoken to. This feature was added after our class pilot test to increase the simulation's immersive feel.

The story itself was designed to be both engaging and authentic in its treatment of how an intern would fit within a corporate environment and the types of argumentative writing tasks they may face. After getting acquainted with the characters and website, students joined a company conference call to be introduced to the team. The "conference call" video feels like a live streaming video, but like all videos is pre-recorded. During the call, students learn from a distraught engineer that Microcore's nanobots, designed to heal cuts in animals, have gotten out of control and have killed a test pig in the process. The remainder of the simulation has students collect and analyze information from interviews, documents, and virtual artifacts and incorporate them into a draft press release (following the Microcore style guide), draft emails to Bob summarizing findings from interviews of the various employees and arguing from data about their role in the accident, explore the crime scene (via an interactive image map), and develop an internal proposal arguing for what went wrong and how to fix it. This "who-dun-it mystery" genre provided a platform for the critical analysis of data and argument writing that are required to help discover what happened and propose a solution. As with ARGs, this is "storytelling as archeology" where players string together the various narrative bits into a coherent whole, which they help create with their assignments. To make sure students didn't waste time on noneducational activities, over $90 \%$ of online and in-class Microcore activities tied directly to critical thinking and argumentative writing practice and instruction.

Instructors are able to discuss professional communication challenges raised in the simulation and strategies for dealing with them. They can do so both "in game" (as puppetmasters) and "out of game" (as classroom instructors). We provide tools to help them with both of these methods that support student reflection. In-class instructional materials and studentcentered activities that coincide with the various simulation days were developed. While these occur in- 
class, instructors and students can still refer to the simulation as if it were a real experience, continuing the TINAG ethos. For example, instructors can discuss with students the need to write a truthful, yet nondamaging press release, will help them with their important work at Microcore.

Instructors can also take on a role similar to an ARG puppetmaster, where they can control certain characters. While not built into our original pilot test, we have since added the ability for teachers to customize and send email messages to individual students or the entire class as if it were from any of the characters. For example, an instructor can write an email from Bob to all students and describe some of the problems with the draft press releases that were submitted by the interns. Our next release will allow instructors to assign a grade (e.g., $\sqrt{ }-,, \sqrt{ }$, or $\sqrt{ }+$ ) to an email submission to Bob, each of which triggers a different response written in the tone of an intern coordinator. Providing critical feedback from a fictional character has the potential to force students to consider the impact of their efforts on future employment and not just their grade on a particular assignment in a class.

Like other experiential simulations, Microcore allows players to take on a role in a complex realworld scenario, provides flexibility in what they do, and provides feedback to players as they progress through the simulation. However, unlike most other experiential simulations, Microcore incorporates several features of ARGs to create a new hybrid that we call a playable case study. As described above, the narrative design allows players to play as themselves in an unfolding mystery, while engaging with fictional characters in a realistic, yet engaging and fun environment and technical platform. Not only does completing assignments affect a player's grade, now it advances a storyline as well. The story and interface are designed to support the TINAG ethos, wherein players can skype with characters, send emails, and in future iterations receive text messages from fictional characters. The multimedia nature of the Microcore intranet keeps things streamlined and organized, while adding to the immersive nature of the simulation. Instructors, conceived of as puppetmasters, also benefit from materials that accompany the simulation, such as discussion questions pertinent to professional communication and ethics, and tools to provide customized feedback in the voice of the characters.

These techniques borrowed from ARGs provide a new level of immersion that we anticipate will improve engagement with the material and the development and transfer of argumentative writing skills in a professional context. However, unlike traditional ARGs, Microcore is designed to be reusable [19] and fit within a formal educational setting including inclass discussions and exercises similar to those conducted around traditional case studies.

\section{Results}

Students expressed appreciation for the way the simulation broke up the normal format of a course, saying things like, "It was interesting to have a different form of media. It at least switched it up and made it interesting." Altogether, there were 10 comments about it being humorous, fun, or interesting, with many students remarking that, especially compared to other classes where they are given a very open prompt, like write an essay in a topic of your choice, the simulation offered something new and interesting. Furthermore, the interface itself was very clear to students who rarely had to ask how to perform certain tasks or where to find content.

Overall, there was strong evidence that students engaged well with the narrative. Students remembered detailed elements of the simulation very clearly. For example, students referred to the characters by name, recounted specific statements made by characters in the interviews, which was worth noting, since students' descriptions of other assignments in their classes were much less detailed (this may speak to McDaniel, Waddill, Finstad, and Bourg's [20] research that interest and narrative improve memory). A number of students also expressed strong feelings about the characters, which shows that they were emotionally engaged with the simulation, even if their views of some of the characters were somewhat negative, such as feelings toward one of the characters, Walter, that was purposefully meant to be a jerk. Interestingly, most students ended up recommending he be fired, even though he was the main Microcore scientist and the company could likely not have succeeded without him. This suggests that the Microcore simulation helped generate emotional responses, which can lead to important in-class discussions and reflections.

Unfortunately, many students perceived the main character - Bob, the intern supervisor - as too silly to fit into what they perceived as an authentic corporate environment. Many of our students in the design team suggested comedy as a way to get students more engaged with the material. These student designers were creative writers, while most of the students in the class were STEM majors, which may account for the different perspective. However, many technical writing students seemed to think that the simulation went too far (both in the script and in the acting), making it seem less useful because, especially compared to how their professor used case studies in the rest of the class, the simulation seemed less like what they would actually experience in the workplace rather than more 
so, despite the more immersive nature of the interface. While at least 8 comments described the simulation as realistic and applicable (with two specifically mentioning that characters' personalities were useful to be exposed to in a corporate setting), there were 13 comments describing the simulation - and Bob in particular - as being exaggerated and unrealistic (e.g., "goofy"). Furthermore, the sometimes derogatory nature of his interactions with the interns (similar to "The Office" boss) was off-putting to these Junior and Senior college students.

For writing assignments, applicability to life outside of school can be crucial to student engagement and motivation, and we have learned that adding too much humor can break the façade for students. This likely reduced student engagement, because one reason narratives captivate human interest is that people experience narrative transport, where they feel they are really part of the story. When the story is unrealistic or unbelievable, it essentially kicks the viewers out of the immersive experience, and they recognize that they are only spectators to an unreal event. This lack of immersion in a story can lead to decreased focus as well as decreased enjoyment [21]. Therefore, the mixed response about whether they felt the narrative was realistic is an important factor for our revision of Microcore. It also illustrates the importance of the overall narrative in supporting TINAG and immersion.

Interacting with characters via videos was another area with mixed results. As expected, character videos helped create an immersive story world where characters "come to life" and are thought of as "real people." However, the ways that students interacted with character videos were not ideal in our pilot test and have been a focus point of our iterative development. In the pilot test, players "interviewed" characters by simply playing a video of the interview, with talk bubbles appearing on the video to show the questions being asked. Even though they were filmed in a webcam style (with fake "static" that looked like a bad connection), the lack of interactivity in the interface led some students to report that the simulation felt like the students were "jumping through the hoops." To address this issue, the current version includes the much more immersive and realistic video call functionality explained before and shown in Figure 2. The "question bank" still limits what players can ask (which is necessary due to the pre-filmed nature of the responses), but it allows them to feel like they have more autonomy. Furthermore, it can support "choose your own adventure" style questions and answers that allow different responses, making it more of an investigation. The filmed segments of the simulation are the least flexible once they are completed, since we can't modify them easily afterward like we can with other content such as emails and files.

When we ran our pilot test, we did not have a teacher backend for the simulation. Our discussions and interview with the instructor helped inform the backend that we have since developed. Specifically, the instructor wanted to easily view student progress and assignments in realtime, which we have since added. Additionally, as described earlier, we have added the ability for a teacher to send messages as any character in the simulation to the entire class or individual students. They can also edit existing messages from characters, which allows us to write key pieces of the message in a character's style, while also allowing teachers to insert specific feedback based on student performance. For example, we have "templates" for pre-created messages to students who did not do well (and those who did well) on an assignment, with a placeholder for the teacher to add her specific comments in a certain place. While we were not able to evaluate this functionality in our pilot test, the instructor indicated interest in this feature. This functionality is critical for a teacher to respond "in-game" as a puppetmaster. If instructors find this too onerous, which is a strong possibility for some instructors, it may work best to have a set of TAs that help grade and give feedback on assignments that are centrally located and funded through proceeds from the sale of the simulation itself. These TAs could be taught the style of the character who is responding, as well as how to apply the grading rubrics and promote the educational outcomes.

The simulation seemed to have presented a clear rhetorical context for students to consider in their written assignments. For example, students' emails and progress reports were directed to a specific character and the internal proposal was written for a specific company and purpose. Too often, when assignments are divorced from context, students struggle to understand their audience and the needed rhetorical stance. While student quality varied, there is no question that the shared rhetorical context provided in the simulation allowed the instructor to clearly articulate proper approaches to writing for the specific audience and characters in the simulation. The common ground that students and the instructor shared from the simulation supported discussions and feedback about audience at a high level of specificity.

Of course, the constrained rhetorical context comes at a cost. In particular, the simulation is necessarily focused on one specific context and area. When students are asked to do more independent research to understand an issue, they may experience a lack of context or clear audience, but they will be able to focus on issues they have a topical interest in, which may 
lead to deeper thinking. Simulations, on the other hand, can provide a clearer rhetorical context, but must attempt to create that engagement via other means, which can be difficult as described above. In our design meetings, we have discussed the potential of having a more open-ended playable case study that allowed students to pursue some of their own interest areas, but within a specific context. For example, we have considered a simulation that lets students choose from among several topic areas, or even propose their own. For example, a writing simulation may ask students to write materials that propose a solution to a problem of their choosing in their local community, all framed within a simulation environment where they must convince a philanthropist to fund their idea. This would make the simulation more akin to an openended ARG than a close-ended ARG [24].

\section{Discussion}

We have learned a great deal from creating the Microcore Playable Case Study that we believe is useful for other educational simulation designers. While this pilot test showed several areas that can still be improved, it illustrates the potential of educational simulations that borrow elements of ARGs to create a more immersive experience. In this section, we reflect on the generalizability of our approach and the design implications of findings. Our hope is to help inspire additional playable case studies that will help better understand their opportunities and limitations.

Playable case studies, like other learning tools, fit certain learning objectives and contexts better than others. Simulations in general, are ideal for situations where players can participate in high-risk situations that allow them to fail or succeed safely. They are also highly scalable compared to some other techniques, though our design with a recommended in-class component places some limits on their scalability. Still, teachers interested in providing, in our case, a clear rhetorical situation and contextualized writing assignments can do so relatively easily with a playable case study. And since the simulation is already in a digital format, it has an even greater potential for online education, where many assignments provide little in the way of an authentic rhetorical situation for students to respond to. Students may be told to write to a certain audience, but without personal interaction with the teacher (who can clarify or even role-play the audience members), it is hard for them to really get to know the imagined audience they are writing to. Therefore, the simulation has significant potential in the ever-growing world of online education. But the success of simulations (like the Harvard Business Simulations) and those reported by Carnes [22] and
Kapp, Blair, and Mesch [23]) rely heavily on the simulation design and levels of immersion.

The structure of the Microcore simulation - days advance when associated tasks are completed, which releases new content on the next day - is fairly flexible, while also providing a concrete and predictable structure that is so important to educational experiences. The transmedia delivery, contained within a single website, makes the simulation more controllable and modular than if content were delivered over many websites and social media channels as is done in most ARGs. It also simplifies funding models where students and instructors gain access to the content after paying for it, as well as simplifying access to the content by schools that use Internet filtering software. Bounding content within a single site has been recommended for educational ARGs for these and related issues [16]. However, this doesn't mean simulations couldn't point to other "in-game" sites (e.g., corporate competitors; fictional news sites; personal character websites) if that content was relatively static and public content.

Our findings emphasized the difficulty of creating a narrative that is both engaging and realistic enough to create true immersion. The "mystery" genre of our Microcore simulation worked well at motivating students to progress. However, our use of humor, or at least the particular brand of humor, only appealed to a subset of our players and seemed to interfere with immersion for some players. While many narrative elements are relatively easy to modify based on feedback (e.g., textual messages, files, and even images), video content is harder to re-film, making it critical that the characters and scripts are well vetted prior to filming. Additionally, as with our simulation, it is likely that others will appeal more to certain students than other. This suggests the importance of finding the right demographic for specific simulation. In our case, we believe the humor and "internship" role will be more appealing to High Schoolers than College Juniors and Seniors, though this remains to be seen.

Creating an immersive experience through the interface development is key. We found ourselves able to present nearly all of the content "in-game" (i.e., part of the fictional world), which helped support the TINAG perspective. For example, the tutorial of how to use the simulation software (e.g., read and post emails, conduct video messages, find files, check off tasks) was built into a welcome message from a character who introduced the players to the corporate intranet. Likewise, assignments were submitted as they would be in a real corporate environment (e.g., an email message). Further integration of text messaging, email that goes to a student's real email account, or 360-degree panorama scenes that facilitate exploration 
of physical places would enhance the level of immersion, though it raises other privacy considerations and we believe should only be offered as an opt-in option. Even classroom discussions of the simulation can be conducted as if it were real (e.g., referencing the characters by name, talking about deadlines as if they were based on the company's schedule) if the instructor is so inclined. The one area that we sacrificed TINAG for the sake of clarity was our "Advance Day" button where we allow players to shift to the next in-game day with the click of a button.

Supporting teachers who utilize playable case studies is an area that is ripe for further research. Our experience with the instructor who used our simulation reiterated the benefits of developing in-class content (e.g., discussion questions, topics to cover, summaries of the narrative in the simulation) that complements the typically out-of-class simulation. Materials that we are developing for High School instructors are much more detailed and tied to specific state and federal learning outcomes, as opposed to the higher-level outcomes associated with argumentative writing in a workplace context that are covered in college technical writing courses. We are anxious to see instructors also utilize the puppetmaster tools, such as sending messages from characters, that we have developed into our most recent version, though also concerned that they will be an added burden for some instructors. As discussed in the prior section, alternative models with centrally located TAs may work better for playable case studies.

While the items discussed so far apply to playable case studies about any content area, it is worth considering writing playable case studies such as Microcore. The authors of Worlds Apart claim that attempts to simulate workplace writing do not really meet their aim because simulations lack the social motives and "local rhetorical complexity" of workplace writing [25]. However, as Boscolo and Gelatti [26] point out, authentic professional contexts are not simply about "practical relevance"; it's largely about teaching students that professional communication is a social act. We do not expect that the line between school and work will become fully blurred through a playable case study, but we do expect that students who adopt TINAG will conceive of writing as social naturally contribute to solving solving real problems in that context.

There are several limitations to our current study, though we believe it generated significant findings that are important to share. It was only conducted in a single class, with a single teacher, using a single example of a playable case study on writing instruction that was in its first version. Some of the findings may be different based on different instructors or a different student body, and we've tried to identify places where we believe that is the case. Additionally, some of our new designs, inspired by the limitations of our original design, have not yet been tested. However, we have explained them here to better articulate the entire design process and highlight not only what works, but also what did not work as well as expected and why. We believe this honest approach is critical so that others will not make some of the same mistakes we have made. Where problems were identified, we tried to articulate our hypothesized solutions to them. Finally, we have tried to define a "playable case study" but only provided a single example in this paper. We hope that our example and description of playable case studies inspires others that are quite different from the one we have developed. Indeed, we are currently working on two other playable case studies that incorporate some different narrative and game mechanics and relate to different content areas. Due to space constraints, we have not discussed these here, but intend to in future publications.

There are several open questions about playable case studies that will require future research. Some related to their design, while others relate to their efficacy. One area that was not addressed at all in the Microcore simulation was student collaboration and coordination on joint work. Collaborative simulations focused on teamwork and project management are popular in business schools, though they do not include the ARG-like interactions that create a playable case study. Future work should consider how to create collaborative playable case studies. For example, can students provide feedback effectively to other students? If so, are there novel techniques that could help them effectively roleplay in the fictional world? How can learning approaches that provide meaningful choices and leverage students' existing interests be incorporated? How can players take on different roles? While we presented many techniques for interacting with the fictional world and characters, there are likely many others that would improve immersion and support TINAG. What is the role of chat bots or virtual reality in playable case studies? While advanced technology is not required to support playable case studies, there will no doubt be ways of improving immersion using new technologies. What is the role of instructors and in-class activities that complement playable case studies? When is it useful to step outside the world (i.e., break TINAG) and when is it useful to stay in-game?

While this study focused on student experience, any teacher knows that how much students like an assignment is not the only factor determining how much they learn. Motivation alone deals with many other factors, including cognitive processes like working memory, social support, and previous 
knowledge. The next phase of this project will include a comparative analysis of students' actual writing products. Authenticity and engagement will be emphasized separately to see how to maximize results. Tests will be administered after completing a playable case study versus a traditional case study to determine how well students understood or remembered the context they were writing about.

\section{Playable Case Study Design Questions}

We are anxious to see others develop playable case studies and in this section offer some practical advice based on our experience. While we have presented some prescriptive advice throughout the paper and shared some of our techniques, here we present questions for designers of playable case studies. We believe this approach may help generate new mechanics that can be used in playable case studies, which we have not yet considered. Each set of questions focuses on one of the following key lenses so critical to playable case studies: Educational Objectives, Narrative, and Interaction Design. We have placed them in that order, since we believe starting with the educational objectives and assignments is the best place to start from given that they are often the least flexible, followed by the narrative and interaction design. However, it is an iterative process that requires designers to keep them all in their head at the same time to some extent.

\section{Educational Objectives}

- What are the core educational objectives of the playable case study?

- What activities and assignments must players perform to achieve those goals?

- How will feedback be provided to players?

- Who will provide feedback and when?

- What educational scaffolding is needed to support player success?

- What artifacts can be created to provide the educational scaffolding?

- What educational scaffolding is best delivered inclass versus online?

Narrative:

- What role will players adopt in the story?

- Will players appreciate their role and be empowered by it?

- What "genre" will the narrative take (e.g., mystery, romance, adventure)? Is it well suited to the educational objectives?

- Does the narrative style (e.g., use of humor) appeal to your core player demographic?

- What characters are needed to advance the story?
- Who will be the guiding character (or characters) that helps players know what to do next?

- How can the story be broken up into discrete modules (e.g., days, chapters)?

- How can the story be told across multiple media channels (e.g., videos, photos, messages, files)?

- How can the story provide players choice and autonomy?

- Is your story authentic enough that players can buy into TINAG?

Interaction Design

- How will players feel immersed?

- How can players be given the illusion that they are in control even when they aren't?

- Can players track their progress?

- How can players interact with characters in authentic, in-game ways?

- Is there an in-game onboarding experience that introduces the platform to players?

- How can the experience bleed into players real lives in authentic ways?

- Have you addressed any problems raised in usability tests of your interaction design?

\section{Conclusion}

We have introduced "playable case studies," a new type of experiential simulation that incorporates components of Alternate Reality Games (ARGs) to provide a playful, yet realistic, entryway into realworld experiences. These playable case studies allow students to participate in an immersive fictional, yet realistic, experience that connects theory and practice and serves as a novel learning platform. The Microcore example, which allows students to develop argumentative writing skills in a realistic and socially embedded rhetorical context, was presented. Students' responses were mostly positive, with some important caveats, mostly related to the specific ways we developed the characters and interaction design, which we have begun to address with improved designs explained in this paper. While we are at the early stages of this work, there is reason to believe that students will continue to respond positively as we learn more about playable case studies and develop better, more immersive ones in the future. We hope our list of questions and discussion of our results will help inspire additional playable case studies.

\section{Acknowledgements}

Funding for part of this work was provided by NSF grant: AISL \#1323306. 


\section{References}

[1] Common Core State Standards Initiative. Common Core State Standards for English language arts \& literacy in history/social studies, science, and technical subjects. Washington, DC: CCSSO \& National Governors Association, 2010.

[2] Wagner, Tony. The global achievement gap: Why even our best schools don't teach the new survival skills our children need-and what we can do about it. Basic Books, 2010 .

[3] McNamara, Billie R. "The Skill Gap: Will the Future Workplace Become an Abyss." Techniques: Connecting Education and Careers 84.5, 2009.

[4] Career Builder Survey, 2014. www.careerbuilder.com/share/aboutus/pressreleasesdetail.as px?sd=4/10/2014\&id=pr817\&ed $=12 / 31 / 2014$

[5] Kolb, David A. Experiential learning: Experience as the source of learning and development. Pearson Education, 2014.

[6] Wouters, Pieter, Christof van Nimwegen, Herre van Oostendorp, \& Erik D. van der Spek. " A meta-analysis of the cognitive and motivational effects of serious games." Journal of Educational Psychology 105.2, 2013..

[7] Sitzmann, Traci. "A meta-analytic examination of the instructional effectiveness of computer-based simulation games." Personnel psychology 64.2, 2011.

[8] Vogel, Jennifer J., David S. Vogel, Jan Cannon-Bowers, Clint A. Bowers, Kathryn Muse, \& Michelle Wright. "Computer gaming and interactive simulations for learning: A meta-analysis." Journal of Educational Computing Research 34.3, 2006.

[9] Girard, C., Jean Ecalle, \& Annie Magnan. "Serious games as new educational tools: how effective are they? A metaanalysis of recent studies." Journal of Computer Assisted Learning 29.3, 2013..

[10] Kozlowski, Steve W.J., \& Eduardo Salas, eds. Learning, training, and development in organizations. Taylor \& Francis, 2009.

[11] Tobias, Sigmund, \& J.D. Fletcher. Computer games and instruction. IAP, 2011.

[12] Gredler, Margaret E. "17. Educational Games and Simulations: A Technology in Search of a (research) Paradigm." Technology 39, 1996, pp. 521-540.

[13] Gredler, Margaret E. "Games and simulations and their relationships to learning." Handbook of research on educational communications and technology 2, 2004.
[14] Bonsignore, Elizabeth, Derek Hansen, Kari Kraus, \& Marc Ruppel. "Alternate reality games as platforms for practicing 21st-century literacies." International Journal of Learning and Media, 2013.

[15] Bonsignore, Elizabeth, Kari Kraus, June Ahn, Amanda Visconti, Ann Fraistat, Allison Druin, \& Derek Hansen. "Alternate reality games: Platforms for collaborative learning." Proceedings of the 10th International Conference of the Learning Sciences, ICLS 2012. Vol. 1. 2012.

[16] Bonsignore, Elizabeth, Derek Hansen, Kari Kraus, Amanda Visconti, June Ahn, \& Allison Druin. "Playing for real: designing alternate reality games for teenagers in learning contexts." Proceedings of the 12th International Conference on Interaction Design and Children. ACM, 2013.

[17] Moseley, Alex. "An Alternate Reality for Education?: Lessons to be Learned from Online Immersive Games." International Journal of Game-Based Learning 2.3, 2012.

[18] Whitton, Nicola. "Alternate reality games for developing student autonomy and peer learning." Proceedings of the LICK Symposium. 2008.

[19] Hansen, Derek, Elizabeth Bonsignire, Marc Ruppel, Amanda Visconti, \& Kari Kraus. "Designing reusable alternate reality games." Proceedings of the SIGCHI Conference. ACM, 2013.

[20] McDaniel, M. A., Waddill, P. J., Finstad, K., \& Bourg, $\mathrm{T}$. The effects of text-based interest on attention and recall. Journal of Educational Psychology, 92.3, 2000.

[21] Green, Melanie C., Timothy C. Brock, \& Geoff F. Kaufman. "Understanding media enjoyment: The role of transportation into narrative worlds." Communication Theory 14.4, 2004, pp. 311-327.

[22] Carnes, M. C. Minds on fire: How role-immersion games transform college. Cambridge, MA, Harvard UP, 2014.

[23] Kapp, K. M., Blair, L., \& Mesch, R. The gamification of learning and instruction fieldbook: Ideas into practice. San Francisco, Wiley, 2014.

[24] Bonsignore, E., Moulder, V., Neustaedter, C., Hansen, D., Kraus, K., \& Druin, A. "Design tactics for authentic interactive fiction: insights from alternate reality game designers." Proceedings of the SIGCHI Conference, ACM, 2014 .

[25] Dias, Patrick, A., Medway, P., and Par, A. Worlds apart: Acting and writing in academic and workplace settings. Routledge, 1999.

[26] Boscolo, P. \& Gelati, C. Best practices in promoting motivation for writing. In S. Graham, C. A. MacArthur, and J. Fitzgerald (Eds.), Best Practices in Writing Instruction (2nd ed.). NY, Guilford, 2013. 